\title{
Die Abwesenheit der Phänomenologie in der deutschen Humangeographie
}

\author{
Jürgen Hasse \\ Institut für Humangeographie, Goethe-Universität Frankfurt, Theodor-W.-Adorno-Platz 6 (PEG), \\ 60323 Frankfurt/M., Germany \\ Correspondence to: Jürgen Hasse (hasse.juergen@t-online.de)
}

Received: 27 December 2016 - Revised: 5 July 2017 - Accepted: 11 July 2017 - Published: 8 August 2017

\begin{abstract}
Kurzfassung. This article is written from the perspective of phenomenology. Its potential gain for a critical human geography is discussed in contrast to the paradigmatic frame of basic assumptions in constructivism. The example of atmospheres will illustrate another theoretical conception of space. In phenomenological view there happens not only a reality of things but also a circum-actuality is not spatially extended like a house or another material objective. Atmospheres are vital qualities (Dürckheim) we feel like a cloud in our sense perception in situations of awareness. This implies the necessity to make a difference between a material body ("Körper") and a felt body ("Leib"). This epistemic knowledge will improve our critique of neoliberal societies, tuned by aestheticisation especially in glamour CBDs of postmodern cities. Finally there is a close link to the work of Michel Foucault, topped off in his The Hermeneutics of the Subject. References to the Critical Theory (Frankfurter Schule) are connected.
\end{abstract}

\section{Kritik am relationalen raum- und akteurs-theoretischen Menschenbild}

Mit dem ab 1970 eingeleiteten Paradigmenwechsel wurde die Humangeographie in eine sozialwissenschaftlich orientierte Raumwissenschaft umgebaut und methodologisch einer Soziologie des Raumes ähnlich gemacht. Die „kritisch geläuterte" Geographie zeichnete sich bis in die 1990er Jahre durch ein geradezu idiosynkratisches Verhältnis gegenüber nicht-relationalen Raum-Begriffen und nicht-konstellationistischen Meta-Theorien aus. Daraus sollte ein denkwürdiges Raum-Verständnis entstehen, wonach der „Zuständigkeitsbereich des Raumbegriffs für die physische Welt nicht überschritten“ (Werlen, 1999:222) werden solle. Das bedeutete die Abkehr von allem, was in seiner Flüchtigkeit und Luzidität weder physisch-materiellen Charakter hat, noch aus dem Kontext menschlichen Handelns heraus zu erklären ist.

Im Zuge eines auch die Wissenschaften erneuernden demographischen Wandels schwächt sich dieser Dogmatismus seit Beginn der 2000er Jahre allmählich ab; vgl. u.a. Dörfler (2015), Kazig (2013 und 2016), Rothfuß (u. a. in diesem Heft), Zahnen (2015), Runkel (2016). Indes darf eine fort- bestehende Macht dieser über lange Jahre eingeschliffenen „Denkstimmung“ (Fleck, 2011) nicht unterschätzt werden. Auch darf und soll nicht übersehen werden, dass es stets beharrende Tendenzen in der Arbeit eher geisteswissenschaftlich orientierter RepräsentantInnen des Faches gegeben hat, die jedoch eher im Schatten des Mainstream wirksam waren; so zur Hermeneutik in der Humangeographie z.B. Jürgen Pohl (vgl. 1986), zur Bedeutung der psychologischen Situiertheit des Menschen in seiner räumlichen Welt Peter Jüngst (vgl. 2000) sowie Oskar Meder und Peter Jüngst (vgl. 2002) oder Autoren, die sich weitgehend aus der Geographie verabschiedet haben (so z.B. der Verfasser). Da insbesondere prekäre Situierungen im Abseits oder Außen einer Disziplin nie allein Folge einer Selbstzuschreibung von Identität sind, sondern ebenfalls Produkt von Fremdzuschreibungen, sind die sich in der Spannung zum vorherrschenden Denken eines Faches entwickelnden Positionen zwangsläufig biographisch „verzeichnet“. Generell wird es vom (subjektiven wie objektiven) Standort eines jeden in „seiner“ (mehr oder weniger ,eigenen“) Community abhängen, ob und inwieweit die folgenden Rekonstruktionen und Positionen auf Zustimmung stoßen oder eher mit kritischer Distanz kommentiert werden. 
Aus der Sicht des Verfassers distanzierte sich die sozialwissenschaftlich erneuerte Humangeographie spätestens seit Beginn der 1990er Jahre von all jenen Theorien, die für den neuen Geist Rückschritt und Moder bedeuteten. Der Ausschluss traf vor allem die sich dem subjektiven Erleben zuwendenden Geisteswissenschaften. Dieses Vergessenmachen hatte zwei Dimensionen. Zunächst galt die Abstandnahme beharrendem geisteswissenschaftlichen Denken innerhalb der Humangeographie. Unter dem Label „Humanistische Geographie“ waren in den 1980er Jahren Impulse eines Anders-Denkens vor allem Anne Buttimer (vgl. 1984) zu verdanken; nachhaltige Resonanz sollten sie indes nicht haben. Im Sinne eines Schneeballsystems sollte aber die Distanz gegenüber außerdisziplinären Denk- und Theorietraditionen aus Erkenntnistheorie und Philosophie, in deren Zentrum die menschliche Subjektivität stand, weit folgenreicher sein. In vielen dieser Theorien spielte der Raum eine wichtige Rolle; beispielhaft sei erinnert an Werke von Otto Friedrich Bollnow (vgl. 1963), Karlfried Graf von Dürckheim (vgl. 2005), Helmut Plessner (vgl. 1980), Erwin Straus (vgl. 1960) und früher schon von Philosophen wie Theodor Lipps (vgl. 1903) oder Johannes Volkelt (vgl. 1905ff). Besonders nachhaltig sollte die Distanzierung gegenüber phänomenologischen Schulen sein, die sich nicht in die Theoriebilder von Materialitäten, relationalen Räumen und intelligibel handelnden Subjekten fügen sollten.

Es galt die Auffassung, dass aus dem Denkraum der Wissenschaft herausgefiltert werden musste, was der Abkehr vom Prinzip des Idiographischen und der Anschauung zuwidergelaufen wäre: Die Stilllegung des Denkens in Kategorien individuellen Erlebens und leiblichen Spürens mitweltlicher Milieus (vgl. auch Plessner, 1980; Heidegger, 1993 und Binswanger, 1993). In der Folge wurde das Subjektive schnell als etwas Individuelles falsch verstanden. Viel zu wenig ist es in Gestalt kollektiver Subjektivität in seiner ganzen erkenntnistheoretischen Reichweite und interdisziplinären $\mathrm{Fa}$ cettenvielfalt dem wissenschaftlichen Denken nützlich gemacht worden. Unter der Führung sozialwissenschaftlicher Theorien verdienten Mitwelten weniger Aufmerksamkeit als systemtheoretisch und konstellationistisch erklärbare Umwelten. Der lebendige und dynamische Fluss ganzheitlichmannigfaltiger Situationen stand diesem Denken ebenso entgegen wie in der Gegenwart der Begriff des Leibes einer wissenschaftssprachlich ubiquitären und höchst kontingenten Körper-Metaphorik.

Auf ihren paradigmatischen Modernisierungswegen hat sich die Geographie besonders in den letzten 20 Jahren von angelsächsischen Diskurs-, Begriffs- und Themen-Moden antreiben lassen. Eine vorschnelle Überanpassung ging oft zulasten einer zukunftsorientierten Nutzbarmachung raumtheoretisch bedeutsamer geisteswissenschaftlicher TheorieTraditionen, deren Vergessen ohnehin seit 1970 weit vorangeschritten war. Die Spuren dieses Vergessens drücken sich in besonderer Weise im aktuellen Raum-Begriff von Raum-Soziologie und Humangeographie aus. Bei Martina
Löw konstituieren sich Räume etwa ,durch (strukturierte) (An)Ordnungen von sozialen Gütern und Menschen an Orten. Räume werden im Handeln geschaffen, indem Objekte und Menschen synthetisiert und relational angeordnet werden“. (Löw, 2001:204). In phänomenologischer Sicht deckt dieses relationale Raum-Denken aber nur bestimmte Klassen von Räumen ab. Danach könnte es gar keine atmosphärisch spürbaren Räume geben, die mit Materialitäten nichts zu tun haben wie zum Beispiel den beengenden leiblichen Raum schneidender Kälte in einem winterlichen Sturm. Der materialistisch-physikalistische Körper-Raum erinnert an Demokrit, der das Wirkliche auf die festen Körper (Atome) begrenzte und alles andere, was man wahrnehmen konnte ,als bloß subjektives Spiel der Sinnlichkeit zur Privatsache der einzelnen Seelen herabsetzte“. (Schmitz, 1980:11). In der Geographie mag die Distanz gegenüber luziden ,herumwirklichen Vitalqualitäten“ (Dürckheim) auch damit zu tun haben, dass sie dem Besonderen, Einmaligen und Idiographischen nahestehen, das seit dem Kieler Geographentag zu den härtesten Idiosynkrasien des Faches gehört.

Während der relationale Raum-Begriff die Stofflichkeit der Dinge präferiert, so das handlungstheoretische Denken den intelligiblen Akteur. Beide Denkwege zusammengenommen, lassen das Zerrbild einer Welt nachdenkender Wesen entstehen, die darüber entscheiden, wie sie sich und die Dinge im Raum platzieren wollen. Diese Fokussierung impliziert notwendige Ausblendungen. Die Idee eines rational handelnden und seine Welt konstruierenden Akteurs verlangt die Tabuisierung - zumindest aber die Marginalisierung - all jener menschlichen Seins-weisen, die sich nicht in die Logik der Fiktion einer anthropologischen brain-maschine fügen wollen: des Zufälligen, Ästhetischen, Performativen, der Macht des Ereignisses, des Sinnlichen, Affektiven und nicht zuletzt des Irrationalen. Mit anderen Worten: Im Schatten der konstruktivistischen Idee eines sich und die Welt ,produzierenden"1 Subjekts wird - der Programmatik kritischer, gegenüber Fragen der politischen Ökonomie aufgeschlossenen Strömungen der Disziplin zum Trotz - die ökonomische, politische und technologische Bedeutung der gefühlsmäßigen sowie irrationalen Seite des Menschen weitgehend übersehen. ${ }^{2}$

\footnotetext{
${ }^{1}$ Eine geradezu ausufernde Rhetorik der „Produktion“ des Raumes geht auf Henri Lefèbvre zurück, der zwischen in seinem neomarxistischen Konzept drei Ebenen der Raumproduktion unterscheidet (eine physische, eine gedankliche und eine gesellschaftliche). Dabei betrachtete er selbst die Ebene des Erlebens noch als eine der Produktion; vgl. Lefebvre (2002).

${ }^{2}$ Auch in der angelsächsischen Debatte um Raum und Gefühl sind Entgrenzungen und Polarisierungen üblich, die einer Wiederaufnahme geisteswissenschaftlicher Traditionen im Wege stehen. Steve Pile gibt 2010 einen Überblick über die humangeographische Forschung zum Verhältnis von Raum und Gefühl im angelsächsischen Sprachraum. Es seien vor allem die Strömungen innerhalb der humanistischen Geographie, die sich auch weiterhin mit der Analyse subjektiver Raumbeziehungen befassen; vgl. Pile (2010:7).
} 
Die folgenden Umrisse phänomenologischen Denkens werden nicht als Vorschlag für einen Ersatz relationalräumlicher wie konstruktivistischer Modelle verstanden, sondern als deren Ergänzung. Mit anderen Worten: Die Relevanz konstruktivistischer Raumkonzepte ist nicht zu bestreiten, nur haben sie ihren spezifischen erkenntnistheoretischen Ort. Es gibt nicht nur Konstruktionen, sondern auch Prozesse der Konstitution. So ließen sich die hier formulierten Vorschläge - soweit dafür (nicht zuletzt emotionale) Bereitschaft besteht - am ehesten in den „Innovationspool“ der sogenannten Neuen Kulturgeographie einfädeln.

\section{Der Beitrag der Neuen Phänomenologie - oder: Die nicht-abstraktionistische Perspektive}

In der Philosophie nimmt sich besonders die Phänomenologie dem Erleben der Welt in der Perspektive der Ersten Person an. Phänomenologie ist Philosophie der Subjektivität und deshalb Philosophie der Mikrologien. Sowenig Subjektivität Solipsismus bedeutet, sowenig führt die erkenntnistheoretische Sensibilität gegenüber dem Detail in die kleinkarierte Haarspalterei. In die Irre muss dagegen jenes SubjektivitätsVerständnis führen, wie es sich (als Ausdruck eines wissenschaftstheoretischen Mainstreams) im Lexikon der Geographie darstellt. Unter dem Stichwort „Subjektivität“ wird dort nämlich allein die „Besonderheit der Erkenntnis eines einzelnen Subjekts“ (Werlen, 2002:311) hervorgehoben. Der methodologische Individualismus steht im krassen Gegensatz zu solchen Theorien, die sich der Rolle des Subjekts in der Gesellschaft bewusst waren und sind. So merkte Adorno an, dass das Subjekt als Individuum, ,nicht weniger in sich gefangen [ist] als in der Allgemeinheit, der Gesellschaft"“. (Adorno, 1980:160). Noch eindrücklicher wird der nur scheinbar rein individuelle Charakter von Subjektivität bei Adorno und Horkheimer als „Pseudoindividualität" desillusioniert; danach seien die Individuen ,gar keine [...], sondern bloße Verkehrsknotenpunkte der Tendenzen des Allgemeinen“ (Adorno und Horkheimer, 1971:139). Selbst die einzig systematisch aufgebaute (Neue) Phänomenologie von Herrmann Schmitz, die auch als allgemeine Erkenntnistheorie ausgearbeitet ist, positioniert die Person zwischen Individuum und Gesellschaft, man könnte auch sagen zwischen individueller und kollektiver Subjektivität. Mit Begriff und Konzept der „gemeinsamen Situation“ bringt Schmitz Herde von Bedeutungen ins Spiel, die gesellschaftlich verortet sind. ${ }^{3}$ Auch

Dabei werden Affekte ,in opposition to cognition, reflexivity, consciousness und humanness" gesehen; ebd.:8. Schon diese Polarisierung ist nicht unstrittig, sind doch Affekte nicht a priori das Andere des Bewusstseins und auch der Reflexivität nicht entgegengestellt. Sie bilden vielmehr ein spezifisches Seins-Milieu, in dem sich Reflexivität anders orientiert als im Metier der Kognitionen.

${ }^{3}$ „Die persönliche Situation ist nicht eine isolierte Angelegenheit des Individuums, sondern eingebettet in gemeinsame Situationen, aus denen sie hervorwächst, die ihr Halt und Tiefe geben, in die sie aber auch hineinwächst.“; Schmitz (1990:76). darin wird deutlich, dass ein allein individualistisch aufgefasster Subjektivitäts-Begriff auf eine politische Desensibilisierung hinausläuft.

Es ist evident, dass der (Human-) Geographie mit der wissenschaftshistorischen Verabschiedung der idiographischen Methode der Sinn für die Mikrologien unterhalb luftiger Abstraktionsbasen metatheoretischer Konstrukte weitgehend verloren gegangen ist. Indes illustrieren große historische Werke zur Ästhetik wie zur Kunstgeschichte den erkenntnistheoretischen Ertrag der Aufmerksamkeit gegenüber dem Banalen und Infra-Normalen für die präzise Analyse rational-irrationaler Wirkungsketten. Auch jede gesellschaftskritische Analyse von Macht und Praktiken der Herrschaft bedürfte im Prinzip einer Archäologie subjektiven Erlebens zum Beispiel von (dissuasiven) Architekturen wie Medien im Allgemeinen. Dies schon deshalb, weil Gefühle die Horte von Bedeutungen sind. Es ist angesichts dessen bemerkenswert, dass gerade die Archäologie innerhalb der gesellschaftswissenschaftlichen Disziplinen die Bedeutung der Gefühle im Leben der Menschen früherer Zeiten zu ihrem Forschungsthema macht, indem sie versucht, die emotionale Bedeutung von Dingen und Plätzen zu rekonstruieren. Sie strebt auch danach, Gefühle in der eigenen emotionalen Beziehung zur Archäologie (Tarlow, 2012:178) aufzuspüren und geht sogar der Frage nach, inwieweit Empathie methodologischen Charakter hat (vgl. ebd.: 179): „Although an awareness of the positioned subjectivity of the archaeologist is important" (ebd.). Es versteht sich von selbst, dass solche Forschung stets mit der Erkundung des Idiographischen beginnt.

Erst kürzlich unterzog David Seamon die historische Abkehr der Humangeographie von der Phänomenologie einer kritischen Analyse. Nach seiner Einschätzung befindet sich die phänomenologisch orientierte Humangeographie seit den 1980er Jahren in einem Schlafzustand. Die seit den 1970er Jahren an der phänomenologisch orientierten humanistischen Geographie formulierte Kritik fasste er unter anderem in den folgenden Punkten zusammen:

- Individualität werde zulasten gesellschaftlicher und ökonomischer Kontexte fokussiert;

- Geschichte und Kultur werden erkenntnistheoretisch marginalisiert;

- Subjektivismus sei eine ,insufficient ontology“;

- Prozesse des placemaking (Heimat) seien im Zeitalter der Globalisierung obsolet geworden (vgl. Seamon, 2014).

Bis heute leidet die Phänomenologie in ihrer sozialwissenschaftlichen und humangeographischen Akzeptanz unter der Hypothek einer gewissen Neutralität gegenüber Forschungsthemen und Fragestellungen vom Format gesellschaftlicher 
und politischer Herausforderungen. Ich komme unter Kapitel 3 auf diesen Vorwurf zurück. ${ }^{4}$

Phänomenologie kann nach Schmitz in der Diskussion von Fragen subjektiven Erlebens hilfreich sein. Sie erhellt Schemata subjektiver Wahrnehmung und kann darüber aufklären, was Akteure der Kulturindustrie warum tun, um Menschen zu kolonisieren und zu Objekten zu machen. Phänomenologie leistet damit einen Beitrag zur Kritik an der Zivilisation (vgl. in diesem Sinne auch Böhme, 2008). Sie verschränkt sich mit der Ethnopsychoanalyse, die erklärt, wie die gesellschaftlich subtil vor sich gehende Unbewusstmachung ins Werk gesetzt wird und mythisch funktioniert. Die Forschungspraxis setzt indes andere Zeichen. Nicht nur, aber besonders im neuerlichen sozialwissenschaftlichen Interesse an Gefühlen ist eine doppelte Ignoranz bemerkenswert. Die erste ist Spiegel einer szientistischen Anglomanie. Danach verdient ein englischsprachiger Aufsatz in einem reviewten Journal mehr Beachtung als ganze philosophiehistorisch als wegweisend geltende Werke in deutscher Sprache. Das ist nicht nur ahistorisch, sondern auch unkritisch. Die zweite Ignoranz ist Ausdruck einer sozialwissenschaftlichen Überheblichkeit gegenüber (insbesondere alten oder älteren) geisteswissenschaftlichen Denktraditionen. So merken Gammerl und Herrn in ihrem „kritischen“ Beitrag zur Stadtforschung über Gefühlsräume und Raumgefühle mit Verweis auf die Theorien von Gernot Böhme und Hermann Schmitz ganz lapidar an, dass ,solche phänomenologischen Entwürfe produktiv weiterzudenken" seien (Gammerl und Herrn, 2015:16). Den jeweils immens umfangreich elaborierten Theorien selbst widmen sie noch nicht einmal rudimentärste Überlegungen.

\subsection{Atmosphären als prädimensionale Räume}

Ich werde in den folgenden beiden Unterkapiteln auf dem Hintergrund der Neuen Phänomenologie von Hermann Schmitz am Beispiel der Atmosphären und der Leiblichkeit kurz illustrieren, welches Erkenntnispotential in der systematischen Reflexion subjektiven Erlebens liegt.

Diese Vorgehensweise will nicht irgnorieren, dass es auch andere phänomenologische Schulen und Denktraditionen gibt, die für eine methodologische Erweiterung der Neuen Kulturgeographie produktiv genutzt werden könnten; z.B. von Martin Heidegger (vgl. 1993), Maurice Merleau-Ponty

\footnotetext{
${ }^{4}$ In der Humangeographie kommt es mitunter zu geradezu absurden (impliziten) Distanzierungen gegenüber jedem Verständnis menschlichen Erlebens, in dem auch nur Spurenelemente von Gefühlen ein Rolle spielen könnten. So definiert das Lexikon der Geographie den Begriff des „Wahrnehmungsraumes“ als „die Menge aller Standorte, über die ein Individuum oder ein Haushalt gewisse Kenntnisse besitzt“.; Brunotte (2002:458). Deutlicher könnten auch die Spuren eines rationalistisch gedeuteten Subjekts nicht werden. In diesem Zerrbild sind es kognitiv geordnete Cluster von Bedeutungen, die Aufmerksamkeit auf etwas lenken und nicht Affekte, schon gar nicht Begehrnisse oder irrationale Dispositionen.
}

(vgl. 1945) incl. der darauf aufbauenden Arbeiten von Bernhard Waldenfels (vgl. 2002), Paul Ricœur (vgl. 1986) und für den Bereich der Fachdidaktik die phänomenologische Philosophie von Eugen Fink (vgl. 1995). Die Fokussierung auf die Neue Phänomenologie von Hermann Schmitz begründet sich (rein subjektiv zunächst) damit, dass der Verfasser seit rund 20 Jahren mit der Rezeption des Systems und seiner Anwendung auf theoretische und empirische Felder der Raumforschung befasst ist; die Auswahl ist aber (in gewisser Weise objektiv) auch darin begründet, dass Schmitz der einzige Phänomenologe ist, der nach 1950 ein System der Philosophie (mit 10 Bänden im engeren Sinne und weiteren 50 ergänzenden Büchern) ausgearbeitet hat, dessen Begrifflichkeit sich nicht zuletzt für die systematische Erforschung von Mensch-Raum-Beziehungen anbietet. Aus der Vielfalt der möglichen Thematisierungen greife ich im Folgenden die Atmosphären heraus, nicht zuletzt deshalb, weil sie selbst räumlichen Charakter haben, wenn sich dieser auch vom vorherrschenden geographischen Raum-Denken unterscheidet. Es sei im Sinne einer hier allein möglichen Randbemerkung auf die theoretisch hohe Kompatibilität der Schmitz'schen Phänomenologie mit dem Denken Peter Sloterdijks (u.a. das Sphären-Projekt, vgl. 1998, 1999, 2004) verwiesen.

Gefühle sind nach Hermann Schmitz ,nicht private Zustände seelischer Innenwelten, sondern räumlich ausgedehnte Atmosphären“. In deren Bann kann man ,im Sinne affektiven Betroffenseins von Gefühlen" leiblich spürbar hinein geraten (Schmitz, 1993:33). Atmosphären lassen sich folglich mit und ohne Gefühl der Betroffenheit wahrnehmen, denn „ob und welche Atmosphäre jemanden ergreift, hängt [...] von seinem jeweiligen leiblichen Befinden als dem Boden seiner spezifischen Resonanz für Atmosphären ab" (ebd.).

Otto Friedrich Bollnow hatte den wichtigen Unterschied zwischen Atmosphären und Stimmungen gemacht. Während Hermann Schmitz Atmosphären auf einer Objektseite verortet, sieht Gernot Böhme sie in einem Dazwischen. Atmosphärische Räume haben keinen relational-räumlichen Charakter, sie sind deshalb auch nicht mit dem Begriff des Körpers kompatibel. Tellenbach beschreibt sie als Umwölkungen (vgl. 1968:111), Sloterdijk als schaumartige Gebilde (vgl. 2004:28). Am weitgehendsten ist der von Schmitz erbrachte Nachweis ihrer Prädimensionalität (vgl. 1988:386f.). Danach gibt es „Volumen ohne Dreidimensionalität“, das heißt prädimensionale Volumen. Diese haben ,nirgends eine Fläche und daher auch keine Kante oder Ecke“ (ebd.:387). In solchen umgebenden Räumen befindet man sich wie ein Taucher im Wasser oder ein Wanderer in der Stille des Waldes. Es ist evident, dass das prädimensionale Raumverständnis gegenüber dem derzeit herrschenden geographischen Raumdenken inkommensurabel ist.

Es gehört zur wissenschaftspsychologischen Selbststabilisierung eines jeden diskursiven Systems, dass es sich gegen irritierende Einflüsse, also theoretisch nicht „passende“ Ideen abschirmen muss (vgl. dazu auch Hasse, 2016). „Alles Erkennen ist ein Prozeß zwischen dem Individuum, seinem 
Denkstil, der aus der Zugehörigkeit zu einer sozialen Gruppe folgt, und dem Objekt.“ (Fleck, 2011:412). ${ }^{5}$ Das musste in den 1960er Jahren angesichts der sich anbahnenden Kritik am traditionellen Denken der Geographie schon Herbert Lehmann zu spüren bekommen haben, und so distanzierte er sich selbst von seinen Bemerkungen zu den Atmosphären einer italienischen Landschaft, weil er wusste, dass der neue Geist seiner auf Anschauung basierenden Erkenntnismethode nicht mehr aufgeschlossen gegenüber stehen würde. Renate Müller merkt an, Lehmann habe offensichtlich sogar dazu geneigt, ,die eigene Betroffenheit zu maskieren, da er wenig Verständnis insbesondere seitens seiner Fachkollegen aus den Naturwissenschaften erwartete und wohl fürchtete, seine fachwissenschaftliche Arbeit in Mißkredit zu bringen“. (Müller, 1986:25). Diese Situation hat sich in der Gegenwart zumindest zugunsten der Atmosphärenforschung verändert (vgl. z.B. Kazig, 2013 und Escher, 2008 sowie Escher et al., 2016).

Die Reduktion des Raum-Begriffs auf körperliche Volumen vereitelt aber nicht nur die theoretische Integration prädimensionaler Räume. Mit dem geographischen RaumDenken sind auch die für jedes detaillierte Verständnis von Atmosphären unverzichtbaren „Halbdinge“ inkompatibel. Böhme spricht sie als die Erzeugenden an. Während Dinge stetig dauern (zumindest in der mittleren Zeit vor der Zersetzung der Stoffe), stellt sich bei Halbdingen wie dem Wind, der Wärme, der Stille oder einem Ton nie die Frage, wo sie gewesen sind, als sie gerade nicht da waren (vgl. auch Schmitz, 1990:215ff.). So mündet die objektlogische Fixierung der Geographie aufs Stoffliche auch in eine erkenntnistheoretische Verbiegung der Atmosphären. Als prominenter Vordenker der Systemtheorie spitzt Niklas Luhmann deren Verkennung zu. Wenn er betont, dass Atmosphäre ,nie der Raum selbst“ sei, weil er als „Medium niemals sichtbar werden kann“ (Luhmann, 1997:181f.), übersieht er, dass Räume im Medium der Atmosphäre spürbar werden und ganz ohne (sichtbare und messbare) Dinge ausgedehnt sind. Die Beispiele der Stille und der ermüdungsbedingten, leiblich spürbaren Schwere belegen das.

Beispiele für Atmosphären des Wetters hatte noch in den 1940er und 50er Jahren Willy Hellpach geliefert. Seine Beschreibungen bedienten sich metaphorischer und synästhetischer Begriffe, mit denen er zum Ausdruck brachte, was zwischen leiblichem Befinden und mitweltlicher ,Vitalqualität“" (Dürckheim, 2005:39 ${ }^{6}$ ) gleichsam in der Schwebe war. So sprach er von bleierner und schwerer Luft der Schneefälle oder von sengender, brütender und stickiger Luft eines heiBen Sommermittags (vgl. Hellpach, 1946:62). Von solchen

\footnotetext{
${ }^{5}$ „Leicht zu bemerken ist, daß, wenn in einer Gruppe von Menschen ein lebhafter Gedankenaustausch stattfindet, in Kürze eine spezielle kollektive Stimmung entsteht, die bewirkt, daß die Menschen Sätze sagen, die sie in anderen Gruppen nicht gesagt hätten."; ebd.

${ }^{6}$ Dürckheim spricht hier auch von einem ,Vitalton“.
}

atmosphärischen Situationen geht ein mitunter machtvoller Einfluss auf das Befinden der in diesen Umwölkungen sich befindenden Menschen aus, den Hellpach mit dem Begriff der „Lufttöne“ ansprach, die es in einer stärkenden und erquicken wie in einer sich weich und schmeichelnd anfühlenden Erlebniswirklichkeit gebe (ebd.: 59). In der ,,animierenden“ Frühherbstluft fühle man ,eine wohlgestimmte Frische und Betätigungslust, sie wirkt wie ,Champagner' [...]" (ebd.: 60). Diese Eigenschaften der Luft kann man nur bedingt sehen. Man kann sie auch nur sehr begrenzt riechen und mit dem Tastsinn kaum erfassen, vielmehr bekommen wir sie zu spüren, wenn auch ganz sicher nicht am Körper. Und so erleben wir das Wetter in einer (Stadt-) Landschaft in einer Weise, die das eigene Selbst stimmt. Deshalb sprach Hellpach auch vom Ergehen, das vom Erleben geprägt werde (vgl. ebd.: 65). Dass schon der Versuch, an solchem, dem sinnlichen und Ästhetischen gegenüber sensiblen Denken theoretisch Gefallen zu finden, wissenschafts-und karrierepolitisch ins Verderben stürzen kann, liegt in der Geographie auf der Hand, fallen solche Synthesen doch seit der Kieler Wende unter die diskreditierende Kritik des „Umweltdeterminismus".

In der Beschreibung von Atmosphären ist der Regress auf Synästhesien fast unvermeidlich, weil es an ihnen ist, ein leiblich spürbares Erleben in einen sprachlichen Ausdruck zu übertragen. In diesem Sinne sprach Lewis Mumford von der „Farbe der Stadt“ (Mumford, 1951:35). Damit umschrieb er eine Atmosphäre und brachte etwas von jenem Ergehen zum Ausdruck, in dem man eine Stadt als Gemengelage von „Vitalqualitäten“ zu spüren bekommt - in diesem Quartier anders als in einem nächsten. Auch der Geomorphologe Herbert Lehmann sprach noch in den 1950er Jahren - mit Bezug auf die seinerzeit schon 30 Jahre zurückliegenden Arbeiten von Johannes Gabriel Granö - von „Wahrnehmungsgehalten“ wie „Gefühlsfarben“ (vgl. Lehmann, 1950:184).

Damit kommt ein Blick auf die sinnliche Seite räumlicher Umgebungen zur Geltung, der nicht Ausdruck von esoterischem Übermut ist. Was man in einer Gegend atmosphärisch zu spüren bekommt, hat den Eindruckscharakter einer Wirklichkeit (im Unterschied zur Realität), deren Milieu wir als Aufenthaltsqualität, Lebensqualität, Wohnqualität und Urbanität erleben.

\subsection{Leib und Körper}

Besonders ausgeprägte Aversionen kultivieren die Sozialwissenschaften - und mit ihnen die Humangeographie - gegenüber der phänomenologischen Kategorie des Leibes. Das ist insofern sogar konsequent, als sich mit der Leiblichkeit eine Seinsweise des Menschen ankündigt, die mit den theoretischen Eckpfeilern der wissenschaftstheoretischen Abstraktionsbasis der Sozialwissenschaften weitgehend inkompatibel ist. Zum einen ist der Leib kein Körper und folglich nichts Materielles. Zum anderen weist er auf Grenzen des Handelns hin. Schließlich ist er dem Irrationalen nahe, erschüttert also 
das Phantasma des intelligiblen Menschen. Die Geographie kann sich jedoch so lange nicht konsequent auf die große erkenntnistheoretisch bedenkenswerte Breite und Differenziertheit von Subjektivität einlassen, wie sie den Menschen als ein intellektualistisch leibloses Wesen denkt. Das Subjekt der Geographie ist ein denkendes und agierendes aber kein fühlendes; es ist ein Körper mit stofflichen Organen und biochemischen Prozessen. Aber es ist kein leibliches Wesen, das von sinnlichen Eindrücken affektiv berührt wird und in der Folge nur möglicherweise rational handelt.

Die Frage nach dem Leib gehört zu den theoretischen Kerndebatten der phänomenologischen Philosophie des 20. Jahrhunderts (vgl. Meyer-Drawe, 2004). Dabei hat Husserls Rede vom Leibkörper einem klaren Verständnis des Leibes im Unterschied zum Körper mehr entgegengestanden als eine nachvollziehbare Differenzierung vermittelt. Auch seine Rede vom Leib als ,merkwürdig unvollkommen konstituiertes Ding“ (Husserl, zit. bei Meyer-Drawe, ebd.: 331) macht die Distanz der Sozialwissenschaften gegenüber dem Begriff des Leibes verständlich. Und wenn der Husserl-Schüler Eugen Fink die Leibhaftigkeit des Menschen als die dunkle Seite seiner Existenz anspricht (ebd.: 332), weckt das eher mystische bis esoterische Assoziationen, baut aber keine Brücken $\mathrm{zu}$ einem geisteswissenschaftlichen Denken, das für die Sozialwissenschaften einen Nutzen verspräche. Diese erkenntnistheoretisch eher verwischten Spuren ziehen sich bis zu Merleau-Ponty und Levinas. Bernhard Waldenfels knüpft hier an und will ausgehend von einer ,leiblichen Responsivität" die Tradition Husserls klärend fortführen.

Hier geht Hermann Schmitz einen anderen Weg. Er will sich nicht in einem „Käfig der Tradition“ verfangen, der sich gegenüber den unwillkürlichen Erfahrungen des täglichen Lebens abgeschottet hat. So setzt er mit dem Aufbau einer Neuen Phänomenologie bei der Ausarbeitung eines systematischen Instrumentariums an, das eine ,Sensibilität für das vermittelt, was unwillkürlich betroffen macht" (Schmitz, 1994:XIII). Damit macht Schmitz zum Gegenstand der Erkenntnistheorie, was in der europäischen Kultur ,sozusagen von Amts wegen den Dichtern übertragen“" war (ebd.).

Schmitz grenzt den Leib vom Körper ab, ohne das zwischen beiden bestehende Wechselwirkungsverhältnis zu verkennen: „Leiblich ist, was jemand in der Gegend (keineswegs, wie z.B. im Blick deutlich wird, immer in den Grenzen) seines materiellen Körpers von sich selbst [...] spüren kann, ohne sich der fünf Sinne [...] und des aus ihrem Zeugnis abgeleiteten perzeptiven Körperschemas (der habituellen Vorstellung vom eigenen Körper) zu bedienen.“ (Schmitz, 2011:5). Im Medium der Leiblichkeit findet sich der Mensch in Situationen, die ihn durch ihre Bedeutungen in spezifischer Weise berühren und im Übrigen für die eine oder andere Handlungstendenz orientieren. Der Leib ist „im Gegensatz zum sicht- und tastbaren Körper kein stetig zusammenhängendes Ganzes, sondern ein Gewoge verschwimmender Inseln“ (ebd.: 8). Eine solche Leibesinsel bildet sich zum Beispiel in der Gegend eines Mückenstichs, kann aber auch atmosphärisch bestimmt sein, wie im Gefühl der Leichtigkeit oder der von Trauer hervorgerufenen abgründigen Schwere. Leibesinseln konstituieren sich als ,absolute Orte" mit prädimensionalem Raum-Charakter. Folglich sind sie „unabhängig von Lage- und Abstandbeziehungen“ (Schmitz, 1998:14). Schmerzherde sind zwar physiologisch begründet und im Körper lokalisiert, spürbar sind sie aber leiblich und nicht körperlich.

Die Alltagssprache steht der Bedeutung des Leib-Begriffs weniger fremd gegenüber als die Sprache der Sozialwissenschaften. Die Rede, wonach man ,,sich in seiner Haut wohlfühlt", meint ja nicht die physische, fleischerne Haut, vielmehr ein umhüllendes Gefühl, wodurch ein aktuelles Befinden gestimmt ist. Und wenn es im 19. Jahrhundert über ein leck geschlagenes Schiff hieß, dass der Schiffer, um „Leib, Schiff und Gut zu erhalten, den Strand suchen“ (Herquet, 1991:30) musste, so ist auch hier mit dem Leib etwas gemeint, das sich nicht durch „Körper“ ersetzen lässt. Die Körper der Ertrunkenen waren ja auch nach dem Scheitern des Schiffes erhalten, nur lebten sie eben nicht mehr. Gänzlich absurd wäre es, anstelle des alten Begriffs „Leibarzt“ nun „Körperarzt“" zu sagen, steckt im Begriff „Leibarzt“ doch die Bedeutung eines spezifisch pathischen Verhältnisses des Kranken oder sich krank Fühlenden zu sich selbst, die sich vom spätmodernen Verständnis von Krankheit sowie der Rolle des Arztes unterscheidet.

Ein sensibles Bewusstsein der Bedeutung des Leibes kennzeichnet auch Norbert Elias als Verfasser der wohl wichtigsten europäischen Zivilisationsgeschichte. Die gesellschaftlich-subkulturelle Einübung in die Sitten des Essens und Trinkens oder des Tanzes (bei Rudolf zur Lippe) wäre nicht annähernd zu verstehen, würde man dieses ganz spezifische Üben als „Einkörperung“ auffassen und nicht als Einverleibung. Auch das (poststrukturalistische) Werk von Michel Foucault muss ohne einen verstehenden Sinn für das Leibliche in seinen zentralen Aspekten unvollständig bzw. „schräg“ verstanden werden. Die im 18. Jahrhundert eingeübte militärische „Kontrolle und Übung der individuellen Körper bis zum Einsatz ausgebildeter Kräfte“ (Foucault, 1994:216) impliziert einen Leib-Begriff, ohne den jeder Sinn fern bleibt. Mehr noch stellt sich die „Hermeneutik des Subjekts", die sich dem Thema der übenden Selbstbildung zuwendet, in ihrem Kern als ein Programm leiblicher Übung heraus: „Sich um sich selbst zu sorgen beinhaltet, daß man seinen Blick umkehrt, daß man ihn von außen - ich möchte fast sagen - nach ,innen“ wendet.“ (Foucault, 2004:26f.). Insbesondere in Gestalt touristischer Exzesse spitzt sich die kulturindustriell formatierte (oft genug ins Absurde entfremdete) Sorge um das eigene Selbst in leiblichen Erlebnisekstasen zu und nicht in körperlichen. Auch wenn der Körper als gestisches Medium narzisstischer Selbstpräsentation ge-, überund umgeformt, beschrieben und verkleidet wird, so zielen all diese ästhetischen Anstrengungen doch auf eine ,gutes“ (oder „cooles“...) Gefühl im Resonanzerleben der sozialen Welt signifikanter Anderer ab. 
Mit dem Körper des Menschen haben spürbare Eindrücke, die wir zum Beispiel in urbanen Welten in dichten Gemengelagen der Menschen und Dinge erleben, nicht viel zu tun. Im Raum der Stadt gibt es zahllose Körper ohne Leib Dinge wie Häuser, Automobile sowie alles, was sich nicht selbst wahrnehmen kann. Aber es gibt keinen Leib ohne Körper. Ein Schmerz mag seinen Grund in einer organischen, also körperlichen Funktionsstörung haben. Zu spüren bekommen wir ihn nicht auf den Displays medizinischer Kontrollinstrumente, sondern am eigenen Leib. Deshalb spricht Gernot Böhme vom Leib als Bewusstseinsform (Böhme, 2014:127ff.).

Das sinnliche Vermögen des Menschen verdankt sich einer situativ immer wieder aufs Neue sich einpendelnden und aktualisierenden Synthese von Körper und Leib. Die Sinnesorgane gehören zum Körper; die sich durch sie vermittelnden Eindrücke berühren uns leiblich. Würden wir sie allein als Sinnes-Reize verstehen, könnten wir sie theoretisch im Gehirn und damit wieder im Körper unterbringen. Der Mainstream des Denkens in den Sozialwissenschaften geht genau so vor.

Erkenntnistheoretisch weitreichende Konsequenzen hat der Gebrauch des Körper-Begriffs in den Sozialwissenschaften nicht zuletzt deshalb, weil er auf meist undeutliche Weise (und von Fall zu Fall) die Bedeutung des Leibes impliziert so zum Beispiel dann, wenn „body“ als ,the , authentic“ location of emotions/affects" (Pile, 2010:11) angesehen wird. So macht die Aussage, ,the body is the site of validation of knowledge" (ebd.), zum Beispiel nur in der Interpretation von „body“ im Sinne von Leib Sinn. Wenn Gefühle schließlich (wie bei Thrift) als etwas Hergestelltes gelten, wird ihre Rolle im menschlichen Leben radikal verkürzt. Pile resümiert: „This engineering metaphor has its own technology: it casts affect as something that can be piped or cabled, rewired, rerouted, re-networked in conscious and intentional way.“ (ebd.:12).

Mit Nachdruck setzt sich in den Sozialwissenschaften eine Körper-Metaphorik durch, die Materialitäten in den Blick nimmt und zu gesellschaftlichen Konstruktionen und Produktionen in Beziehung setzt. Die Entdifferenzierung des Körper-Begriffs von dem des Leibes spiegelt im deutschsprachigen Wissenschafts-Diskurs eine weit fortgeschrittene Anglomanie wider. Auf der anderen Seite weisen die angelsächsischen „Embodiment“-Diskurse (vgl. Gallagher, 2005 und 2012) Wege der Differenzierung phänomenologischer Sensibilitäten gegenüber dem, was als Mitwelt über den Begriff der Umwelt hinausgeht, ohne dessen Relevanz zu entwerten.

\section{Optionen der Kritik}

Bis heute sind innerhalb der Sozialwissenschaften wie im Mainstream einer konstruktivistisch orientierten Humangeographie Fluchtbewegungen vor einer solipsistisch erscheinenden phänomenologischen Forschung sehr lebendig. Sol- che Distanzierungen werden aber auch von der Phänomenologie selbst gefördert. Wenn Relph eine Stärke der Phänomenologie in der Geographie darin sieht, dass sie uns zeige, was ist ${ }^{7}$, so scheint damit in der Tat eine solipsistische Seite auf, die Bezüge zur Methodologie der Sozialwissenschaften zerbricht. In der Folge sieht sich die Phänomenologie insbesondere im kritischen Fokus der Sozialwissenschaften dem Vorwurf a-politischer Selbstsituierung ausgesetzt.

Im konstruktivistischen Blick der Sozialwissenschaften auf Atmosphären liegen aber auch Optionen der Kritik. ${ }^{8}$ In deren Fokus gelangen jene systemischen Interessen - allzumal in der Kulturindustrie - die im Gefühlsmedium atmosphärischer Umwölkungen manipulative Macht entfalten, um einen Mehrwert durch die Herrschaft über die Affekte und Begehren der Menschen generieren zu können. So ist das konstruktivistische Denken - wie wir es im Prinzip schon seit Platon kennen - zum Beispiel in der Analyse von Kaufhausatmosphären oder liturgischer Inszenierungen im sakralen Raum geradezu unverzichtbar. In der Frage der mikrologischen Funktionsweisen solcher Suggestionen wie der Autorenschaft affektuell-immersiver Zumutungen bietet die kritische Rekonstruktion affizierender Programme klärende Aufschlüsse.

In der Paarung mit der Großen Erzählung vom ubiquitären Akteur limitieren sich die potentiellen Erträge dieses Denkens wiederum. Der Adressat von Gefühls-Appellen ist ja gerade nicht in der Akteurs-Klasse weitgehend kognitiv handelnder Subjekte zu finden, vielmehr jenes affektiv und irrational lenkbare Objekt-Subjekt, das schon im Zentrum der „Kulturindustrie“ von Adorno und Horkheimer (vgl. 1971) stand und sich bis in die Gegenwart als „Partner“ politischer und massenmedialer Manipulationen immer wieder als nützlich erweist. Patheure reflektieren ihr Verhalten nicht in fortlaufenden Evaluationsschleifen, um es im Sinne intelligibler Selbstzertifizierung rational zu legitimieren. Patheure lassen sich von einem Strom der Affekte in Situationen mitnehmen. Solange das situativ immer wieder um seine Aufklärung betrogene Subjekt (-Objekt) nicht in die kritische Analyse gesellschaftlicher Verhältnisse einbezogen wird, bleiben wichtige dispositive Machtbündel (Foucault), deren Einflüsse die Gesellschaft maßgeblich verändern, außerhalb des Blickfeldes. Die „mythische Verbiegung“ (Barthes, 2010:277) des Subjekts zum Akteur hat deshalb auch einen massiv gegenaufklärerischen Effekt. Dieser besteht in einer Verharmlosung bis Verdeckung der Virulenz politischer und ökonomischer Dispositive der Macht.

\footnotetext{
${ }^{7}$ „It is the attempt to see clearly what there is"; Relph (1981:177).

${ }^{8}$ „Kritik“ verstehe ich hier diesseits ideologischer Denkstimmungen (Fleck) mit Foucault ,als die kritische Haltung, die man im Abendland als besondere Haltung neben dem großen historischen Prozeß der Regierbarmachung der Gesellschaft auftauchen sieht."; Foucault (1992:16f).
} 
Gerade die kulturindustriell überschriebenen Gesichter der Metropolen sind mit Atmosphären gesättigt. Die Menschen könnten das affizierende Theater postmoderner Kaufhausarchitektur als Angriff auf ihre Autonomie besser und leichter „,erkennen“, wenn sie eine Sensibilität für die Schnittstelle von leiblicher Intelligenz und analytisch-intelligentem Denken (vgl. dazu auch Schmitz, 2010:86ff.) übend alphabetisieren würden. Zum einen sind sie sich (als Akteure) dessen bewusst, was sie tun oder lassen. Zum anderen werden sie aber auch in einem halbbewussten Sinne von einem Geschehen getragen. Und so nehmen uns Atmosphären in einem hoch manipulativen Sinne oft in eine Welt der Trugbilder und Einbildungen mit. Phänomenologie strebt an diesem Punkt nach Zuwächsen an Kritik durch Selbstkritik: „Der Fortschritt besteht darin, immer genau zu merken, was merklich ist. Phänomenologie ist ein Lernprozess der Verfeinerung der Aufmerksamkeit und Verbreiterung des Horizonts für mögliche Annahmen." (Schmitz, 2009:14).

Ein ganzer Boom affizierender Stimmungsmächte bedient sich der Instrumente der Verführung und nicht der des rationalen Argumentes. Die kolonisierenden Kalküle gehen auf, wenn das Wollen unterströmt ist und die Menschen glauben, dass sie wollen, was sie doch nur sollen. Darin lag schon ein zentrales Thema der Dialektik der Aufklärung bei Adorno und Horkheimer (vgl. 1971). Mit dieser Entfremdung öffnet sich eine Schere: Je größer das Unbewusste des eigenen Selbst systemisch gemacht wird (vgl. in diesem Sinne Erdheim und Nadig, 1994), desto kleiner wird das Vermögen, über Gefühle und ihre Wirkung treffend sprechen zu können. Und so liegt es auf der Hand, dass sich ein Schwund an Selbstbewusstsein als Ressource der Fremdbestimmung anbietet. Insbesondere nach dem Zweiten Weltkrieg wurden die Medien der Verführung (vgl. in diesem Sinne Baudrillard, 1983) immens pluralisiert und radikalisiert. Damit vergrößerte sich auch die Kluft zwischen den Dispositiven der Macht ästhetizistischer Akteure zum einen, und den individuellen Technologien des Selbst (vgl. Foucault, 1993) zum anderen.

Die Wissenschaften können diese Entfremdung der Subjekte nur bedingt verlangsamen. Zum einen sind sie den Mikrologien des affektiven Erlebens gegenüber weitgehend uninteressiert. Zum anderen haben sie sich eine Sprache gegeben, die vornehmlich sie selbst nur verstehen. In ihrer Abstraktheit vermag sie die Vitalität des sinnlichen und leiblichen Erlebens kaum zu erfassen. Die Konjunktur dieser paradigmatischen Sichtweise liegt zu guten Teilen an der Mode des Konstruktivismus, wonach Gefühle generell als gesellschaftlich hergestellt gelten. So richtet sich das wissenschaftliche Interesse nur noch wenig auf das Verstehen des sinnlichen und leiblichen Erlebens von städtischen Events, Fassaden, Baustoffen, ästhetischen Illuminationen oder suggestiven Klangwelten. Es konzentriert sich vielmehr (konstruktivistisch) auf die Analyse der Übertragung von ErlebnisErwartungen.
Indes bietet die subjektive Einlassung empirisch forschender Subjekte in immersive wie dissuasive Milieus einen Weg der wahrnehmenden Erfassung dessen, was affiziert, berührt, erschrickt, die Aufmerksamkeit lenkt, das aktuelle So-Sein stimmt und von sich selbst ablenkt. An einer Reihe von Beispielen habe ich kürzlich methodologisch und praktisch gezeigt, in welcher Weise „Autopsien“ mitweltlichen Erlebens empirisches Rohmaterial für die systematische Ausdeutung subjektiver Beziehungen zur am eigenen Leibe erfahrenen (räumlichen, sozialen, technischen, artifiziellen, performativ sich verändernden etc.) Welt liefern kann. Im Sinne einer lebenspraktischen (wie forschungsmethodisch gewendeten) Schärfung der Aufmerksamkeit und Differenzierung von Vermögen der sprachlichen Explikation situativen Erlebens mündet die „Sorge um das eigene Selbst“ (i.S. von Foucault, vgl. oben) in das Bemühen um Mikrologien räumlichen Erlebens. Die Orte und Anlässe solcher Selbstgewahrwerdung befinden sich in der alltäglichen Welt - im angreifenden Gefühl einer andrängenden Windböe (vgl. dazu Hasse, 2017a:173-227) oder den Atmosphären unterschiedlichster Märkte (vgl. Hasse, 2017b).

Ein gemeinsames Merkmal des Sloterdijk'schen Plädoyers für das Üben (vgl. 2009) und der Foucault'schen Hermeneutik des Subjekts (vgl. 2004) liegt in der Steigerung der Aufmerksamkeit gegenüber dem eigenen Selbst, bzw. seinem Sich-Finden in Situationen. Die nachdenkende Durchquerung von Geschichten subjektiven So- und Mit-Seins eröffnet - gleichsam vom affizierenden Ende atmosphärischer Machtkalküle mit dem Ziel der Instrumentalisierung der Menschen nach ökonomischen oder ideologischen Interessen - profunde Ansatzpunkte für die Formulierung einer konkreten Kritik gesellschaftlicher Verhältnisse, die nicht in abstrakten Systemen identifiziert werden, sondern an dem, was davon spürbar wird.

Datenverfüg barkeit. Für diesen Artikel wurden keine Datensätze genutzt.

Interessenkonflikt. Der Autor erklärt, dass kein Interessenkonflikt besteht.

Danksagung. Der Verfasser dankt den GutachterInnen für ihre hilfreichen Anmerkungen im Rahmen des Reviewverfahrens.

Edited by: Benedikt Korf

Reviewed by: two anonymous referees 


\section{Literatur}

Adorno, T. W.: Stichworte. Kritische Modelle 2 (zuerst 1969), Suhrkamp Verlag, Frankfurt/Main, 1980.

Adorno, T. W. und Horkheimer, M.: Kulturindustrie. Aufklärung als Massenbetrug (zuerst 1947), in: Dialektik der Aufklärung, Herausgeber: Adorno, Th. W. und Horkheimer, M., Verlag S. Fischer, Frankfurt/Main, 108-150, 1971.

Barthes, R.: Mythen des Alltags. Aus dem Französischen von Horst Brühmann, Suhrkamp Verlag, Frankfurt/Main, 2010.

Baudrillard, J.: Laßt euch nicht verführen!, Merve Verlag, Berlin, 1983.

Binswanger, L.: Ausgewählte Werke. Bd. 2.: Grundformen und Erkenntnis menschlichen Daseins, Herausgeber: Herzog, M. und Braun, H.-J., Asanger Verlag, Heidelberg, 1993.

Böhme, G.: Phänomenologie als Kritik, in: Neue Phänomenologie zwischen Praxis und Theorie. Festschrift für Hermann Schmitz, Herausgeber: Großheim, Michael, Verlag Karl Alber, Freiburg und München, 21-36, 2008.

Böhme, G.: Bewusstseinsformen, Verlag Wilhelm Fink, München, 2014

Bollnow, O. F.: Mensch und Raum, Stuttgart u.a., Verlag W. Kohlhammer, 1963.

Brunotte, E., Gebhardt, H., Meurer, M., Meusburger, P., and Nipper, J. (Hrsg.): Lexikon der Geographie (vier Bände), Band 3, Spektrum Verlag, Heidelberg und Berlin, 2002.

Buttimer, A.: Ideal und Wirklichkeit in der angewandten Geographie, Verlag, Kallmünz/Regensburg, 1984.

Dörfler, T.: Der Raum ist der Freund des Seins. Bachelards Poetik des Raumes als Anstoß zu einer neuen Theorie des Raumes“, in: sub/urban. Zeitschrift für kritische Stadtforschung, 3, 95-108, 2015.

Dürckheim, Graf K. von: Untersuchungen zum gelebten Raum (zuerst 1932), Herausgeber: Hasse, J. (mit Einführungen von Hasse, J., Janson, A., Schmitz, H. und Schultheis, K.), in: Natur - Raum - Gesellschaft, Bd. 4, Selbstverlag Institut für Didaktik der Geographie, Frankfurt/Main, 2005.

Erdheim, M. und Nadig, M.: Psychoanalyse und Sozialforschung, in: Psychoanalyse und Unbewußtheit in der Kultur, Herausgeber: Erdheim, M., Suhrkamp Verlag, Frankfurt/Main, 61-82, 1994.

Escher, A.: Die Atmosphäre des orientalischen Bazars: in: Stadt und Atmosphäre, Die alte Stadt, 35, Heft 2, Herausgeber: Hasse, J., 161-174, 2008.

Escher, A., Karner, M., Kerz, Chr., Rapp, H. und Sommerlad, E.: The atmospheric grid of cruising on the seas, Erdkunde, 4, 313321, 2016.

Fink, E.: Grundphänomene des menschlichen Daseins, Alber Verlag, Freiburg und München, 1995.

Fleck, L.: Schauen, Sehen, Wissen (zuerst 1947), in: Denkstile und Tatsachen, Gesammelte Schriften und Zeugnisse, Herausgeber: Werner, S. und Zittel, C., Suhrkamp Verlag, Berlin, 390-418, 2011

Foucault, M.: Was ist Kritik? (zuerst 1978), Merve Verlag, Berlin, 1992.

Foucault, M.: Technologien des Selbst (zuerst 1982), in: Technologien des Selbst, Herausgeber: Luther, H. M., Gutman, H., and Hutton, P. H., Verlag S. Fischer, Frankfurt/Main, 24-62, 1993.

Foucault, M.: Überwachen und Strafen. Die Geburt des Gefängnisses (zuerst 1975), Suhrkamp Verlag, Frankfurt/Main, 1994.
Foucault, M.: Hermeneutik des Subjekts (zuerst 1981/82), Suhrkamp Verlag, Frankfurt/Main, 2004.

Gallagher, S.: How the Body Shapes the Mind, Oxford University Press, Oxford, 2005.

Gallagher, S.: Phenomenology, Palgrave Macmillan, London, 2012.

Gammerl, B. und Herrn, R.: Gefühlsräume - Raumgefühle. Perspektiven auf die Verschränkung von emotionalen Praktiken und Topografien der Moderne, u b / u r b a n . zeitschrift für kritische stadtforschung, Band 3, Heft 2, 7-22, 2015.

Hasse, J.: Wissenschaft und Mythos. Die Macht der Verführung durch abstraktionistische und verschleiernde Theoreme, in: VIII. Jahrbuch für Lebensphilosophie. Kritik und Therapie wissenschaftlicher Unvernunft, Albuena Verlag, München, 67-88, 2016.

Hasse, J.: Die Aura des Einfachen. Mikrologien räumlichen Erlebens, Band 1, Alber Verlag, Freiburg und München, 2017a.

Hasse, J.: Märkte und ihre Atmosphären. Mikrologien räumlichen Erlebens, Band 2, Alber Verlag, Freiburg und München, im Druck, 2017b.

Heidegger, M.: Sein und Zeit (zuerst 1927), Niemeyer, Tübingen, 1993.

Hellpach, W.: Sinne und Seele. Zwölf Gänge in ihrem Grenzdickicht, Ferdinand Enke Verlag, Stuttgart, 1946.

Herquet, K.: Die Insel Borkum in kulturgeschichtlicher Hinsicht. Mit einer Karte von 1713 (zuerst 1886), Verlag Schuster, Leer, 1991.

Jüngst, P.: Territorialität und Psychodynamik. Eine Einführung in die Psychogeographie, Psychosozial-Verlag, Gießen, 2000.

Kazig, R.: „Einkaufsatmosphären. Eine alltagsästhetische Konzeption", in: Perspektiven sozialwissenschaftlicher Konsumforschung, Herausgeber: Schmid, H. und Gäbler, K., Steiner Verlag, Stuttgart, 217-232, 2013.

Kazig, R.: Die Bedeutung von Alltagsästhetik im Kontext der Polarisierung und Hybridisierung von Städten - eine Spurensuche, in: Fraktale Metropolen. Stadtenwicklung zwischen Devianz, Polarisierung und Hybridisierung, Herausgeber: Weber, F. and Kühne, O.: Springer VS, Wiesbaden, 215-229, 2016.

Lefebvre, H.: Die Produktion des Städtischen Raums (zuerst 1977), AnArchitektur, Nr. 01-03, 94-20, Juli 2002.

Lehmann, H.: Die Physiognomie der Landschaft, Studium Generale, 182-195, 1950.

Lipps, T.: Ästhetik. Psychologie des Schönen und der Kunst, 2 Bände, Verlag von Leopold Voss, Leipzig und Hamburg, 1903.

Löw, M.: Raumsoziologie, Suhrkamp Verlag, Frankfurt/Main, 2001.

Luhmann, N.: Die Kunst der Gesellschaft, Suhrkamp Verlag, Frankfurt/Main, 1997.

Meder, O. und Jüngst, P.: Psychodynamik, Machtverhältnisse und Territorialität in "einfachen" und frühen staatlichen Gesellschaften, Überlegungen und Thesen, Univ.-Bibliothek, Kassel, 2002.

Merleau-Ponty, M.: Phénoménologie de la perception, Gallimard, Paris, 1945.

Meyer-Drawe, K.: Stichwort „Leib“, in: Wörterbuch der phänomenologischen Begriffe, Herausgeber: Vetter, H., Verlag Meyner, Hamburg, 331-337, 2004.

Müller, R.: Die Physiognomie der Landschaft als Thema geographischer Forschung bei Herbert Lehmann, in: Herbert Lehmann. Essays zur Physiognomie der Landschaft, Herausgeber: Krenz- 
lin, A. und Müller, R., Erdkundliches Wissen, Heft 83, Wiesbaden, 7-26, 1986.

Mumford, L.: Megapolis. Gesicht und Seele der Gross-Stadt (zuerst 1945), Bauverlag, Wiesbaden, 1951.

Pile, S.: Emotions and affect in recent human geography, in: Transactions of the Institute of British Geographers, 35, 5-20, 2010.

Plessner, H.: Gesammelte Schriften III. Anthropologie der Sinne, Suhrkamp Verlag, Frankfurt/Main, 1980.

Pohl, J.: Geographie als hermeneutische Wissenschaft. Ein Rekonstruktionsversuch. Münchner Geographische Hefte 52, Kallmünz, 1986.

Relph, E.: Rational Landscapes and Humanistic Geography, Croom Helm, London, 1981.

Ricœur, P.: Die lebendige Metapher, Aus dem Französischen übersetzt von Rainer Rochlitz, Wilhelm Fink Verlag, München, 1986.

Runkel, S.: Zur Genealogie des Atmosphären-Begriffs. Eine kritische Würdigung der Ansätze von Hermann Schmitz und Gernot Böhme, in: Atmosphären des Populären II. Perspektiven, Herausgeber: Wünsch, U., Projekte, Protokolle, Performances, Personen, Posen, Uni-Edition, Berlin, 20-39, 2016.

Schmitz, H.: Neue Phänomenologie, Bouvier Verlag, Bonn, 1980.

Schmitz, H.: System der Philosophie. Band III: Der Raum. Teil 1: Der leibliche Raum (zuerst 1967), Bouvier Verlag, Bonn, 1988.

Schmitz, H.: Der unerschöpfliche Gegenstand, Bouvier Verlag, Bonn, 1990 .

Schmitz, H.: Gefühle als Atmosphären und das affektive Betroffensein von ihnen, in: Zur Philosophie der Gefühle, Herausgeber: Fink-Eitel, H. and Lohmann, G., Suhrkamp Verlag, Frankfurt/Main, 33-56, 1993.

Schmitz, H.: Neue Grundlagen der Erkenntnistheorie, Bouvier Verlag, Bonn, 1994.

Schmitz, H.: Der Leib, der Raum und die Gefühle, Edition tertium, Ostfildern, 1998.
Schmitz, H.: Kurze Einführung in die Neue Phänomenologie, Verlag Karl Alber, Freiburg und München, 2009.

Schmitz, H.: Bewusstsein, Verlag Karl Alber, Freiburg und München, 2010.

Schmitz, H.: Der Leib, De Gruyter, Berlin und Boston, 2011.

Seamon, D.: Lived Emplacement and the Locality of Being. A Return to Humanistic Geography? in: Approaches to Human Geography, 2nd edition, Herausgeber: Seamon, D., Stuart Aitken and Gill Valentine, London, 2014.

Sloterdijk, P.: Sphären (I - III), Suhrkamp Verlag, Frankfurt/Main, 1998, 1999, 2004.

Sloterdijk, P.: Du mußt dein Leben ändern, Suhrkamp Verlag, Frankfurt/Main, 2009.

Straus, E.: Psychologie der menschlichen Welt. Gesammelte Schriften, Springer Verlag, Berlin u.a., 1960.

Tarlow, S.: The archaeology of emotion and affect, Annu. Rev. Anthropol., 41, 169-185, 2012.

Tellenbach, H.: Geschmack und Atmosphäre, Otto Müller Verlag, Salzburg, 1968.

Volkelt, J.: System der Ästhetik (drei Bände), Becksche Verlagsbuchhandlung, München, 1905ff.

Waldenfels, B.: Bruchlinien der Erfahrung, Suhrkamp Verlag, Frankfurt/Main, 2002.

Werlen, B.: Zur Ontologie von Gesellschaft und Raum. Sozialgeographie alläglicher Regionalisierungen, Steiner Verlag, Stuttgart, 1999.

Werlen, B.: „Subjektivität“, Stichwort in: Lexikon der Geographie, Herausgeber: Brunotte, E., Gebhardt, H., Meurer, M., Meusburger, P. und Nipper, J., Bd. 3, Spektrum Verlag, Heidelberg und Berlin, 311-312, 2002.

Zahnen, B.: Tragweiten geographischen Denkens, Passagen Verlag, Wien, 2015. 